\title{
Adiabatic Mach-Zehnder Interferometry on a Quantized Bose-Josephson Junction
}

\author{
Chaohong Lee* \\ Nonlinear Physics Centre and ARC Centre of Excellence for Quantum-Atom Optics, \\ Research School of Physical Sciences and Engineering, Australian National University, Canberra ACT 0200, Australia
}

(Received 15 March 2006; published 10 October 2006; corrected 13 October 2006)

\begin{abstract}
We propose a scheme to achieve Mach-Zehnder interferometry using a quantized Bose-Josephson junction with a negative charging energy. The quantum adiabatic evolution through a dynamical bifurcation is used to accomplish the beam splitting and recombination. The negative charging energy ensures the existence of a path-entangled state which enhances the phase measurement precision to the Heisenberg limit. A feasible detection procedure is also presented. The scheme should be realizable with current technology.
\end{abstract}

DOI: 10.1103/PhysRevLett.97.150402

Quantum interference, one of the most fundamental and challenging principles in quantum mechanics, forms the basis of high-precision measurement and quantum information processing. A basic device capable of performing high-precision measurements is an analogue to the optical Mach-Zehnder interferometer, in which an incoming wave is divided into two parts by a 50:50 beam splitter and then the two parts are recombined by another beam splitter. Conventional Mach-Zehnder interferometry utilizing single-particle states can only reach the standard quantum limit or the shot noise limit on the measurement precision [1]. However, it has been demonstrated that many-body quantum entanglement, such as the photon polarization entanglement [2] and the trapped-ion internal entanglement [3], can enhance the measurement precision to the so-called Heisenberg limit [1] posed by the Heisenberg uncertainty principle. The application of spin entanglement in interferometry has also been discussed [4].

Given the well-developed techniques in preparing and manipulating atomic Bose-Einstein condensates, the interferometric schemes based upon ultracold atoms stimulate great interests. Using spatially separated condensates [5], condensates trapped within double-well potentials [6], and classical Josephson arrays of tunneling coupled condensates [7], the atomic coherence and interference have been demonstrated. All these experiments have utilized the macroscopic quantum coherence which is well described within the mean-field theory. The many-body nature becomes significant for strongly correlated atoms and some many-body quantum effects including quantum squeezing [8], quantum entanglement [9], and quantum phase transition [10] have been explored. These effects lie beyond the reaches of the mean-field theory.

On the other hand, due to the $s$-wave scattering dominating the ultracold collisions, the nonlinear Kerr effect is intrinsic to the atomic condensates. It has been shown that the nonlinear interaction brings a number of novel phenomena [11] including wave mixing [12], soliton [13], dynamical bifurcation [14], and chaos [15]. In this Letter we will demonstrate how the combination of nonlinear and many-body quantum effects can be used to realize a
PACS numbers: 03.75.Dg, 03.75.Gg, 39.20.+q

Heisenberg-limited Mach-Zehnder interferometry with Bose condensed atoms.

In this Letter, in the frame of fully quantized theory, we propose and analyze a practical scheme of a Mach-Zehnder interferometry with a Bose-Josephson junction. The beam splitters are realized by the quantum adiabatic processes through the dynamical bifurcation. To ensure the existence of a path-entangled state, which enhances the phase measurement precision to the Heisenberg limit, the charging energy is chosen to be negative values. We also discuss a feasible procedure for detection and the experimental realization. The proposed interferometry scheme can operate for large particle numbers $\left(\sim 10^{3}\right)$, whereas the schemes of photons [2] and trapped ions [3] can only reach the order of 10 particles. Reduced influence of the environment and a simple detection procedure are advantages of this scheme.

We consider an ensemble of $N$ Bose condensed atoms confined in a double-well potential [or $N$ two-level Bose condensed atoms confined in a single-well potential with laser (or radio frequency) coupling between the two involved hyperfine levels]. Under the condition of tight binding, the system obeys a two-mode Hamiltonian,

$$
H=\frac{\delta}{2}\left(n_{2}-n_{1}\right)-\frac{T}{2}\left(a_{2}^{+} a_{1}+a_{1}^{+} a_{2}\right)+\frac{E_{C}}{8}\left(n_{2}-n_{1}\right)^{2} .
$$

Here, $a_{j}^{+}, a_{j}$, and $n_{j}=a_{j}^{+} a_{j}(j=1,2)$ denote the creation, annihilation, and particle number operators for the $j$ th mode, respectively. This Hamiltonian describes a quantized Bose-Josephson junction with an imbalance $\delta$, an intermode coupling $T$, and a charging energy $E_{C}$. The values of $\delta, T$, and $E_{C}$ are controlled by the potential asymmetry (or the internal energy difference), the tunneling strength between two wells (or the Rabi frequency for coupling fields), and the $s$-wave scattering lengths, respectively $[8,9,14-16]$. Regarding all atoms as spin- $1 / 2$ particles, one can define the angular momentum operators as $J_{x}=\left(a_{2}^{+} a_{1}+a_{2} a_{1}^{+}\right) / 2, \quad J_{y}=i\left(a_{2}^{+} a_{1}-a_{2} a_{1}^{+}\right) / 2, \quad$ and $J_{z}=\left(a_{2}^{+} a_{2}-a_{1}^{+} a_{1}\right) / 2$. Thus, $H=\delta J_{z}-T J_{x}+E_{C} J_{z}^{2} / 2$ and an arbitrary state can be expanded as a superposition of 
different states $\left|J=N / 2, J_{z}=M\right\rangle$ with $M=-N / 2$, $-N / 2+1, \ldots,+N / 2$.

The ground states of the quantized Bose-Josephson junction sensitively depend on the parameters. For a symmetric junction $(\delta=0)$, in the strong coupling limit $\left(T /\left|E_{C}\right| \gg 1\right)$, the ground state is a $\mathrm{SU}(2)$ spin coherent state $\exp \left(i \phi J_{z}\right) \exp \left(i \theta J_{y}\right)|N / 2,+N / 2\rangle$ with $\phi=0$ and $\theta=\pi / 2$. In the weak coupling limit $\left(T /\left|E_{C}\right| \ll 1\right)$, the ground state relies on $E_{C}$. If $E_{C}>0$, the ground state approaches to $(|N / 2,-1 / 2\rangle+|N / 2,+1 / 2\rangle) / \sqrt{2}$ for odd $N$ or $|N / 2,0\rangle$ for even $N$, when $T \rightarrow 0$. If $E_{C}<0$, the ground state $|0\rangle$ and the first excited state $|1\rangle$ become degenerate when $T \rightarrow 0$, as shown in Fig. 1(a). The critical value between nondegeneracy and degeneracy corresponds to a classical Hopf bifurcation from single-stability to bistability [14]. With $T=0$, these two states are the lowest spin state $|N / 2,-N / 2\rangle$ and the highest spin state $|N / 2,+N / 2\rangle$, respectively. However, the degeneracy between $|0\rangle$ and $|1\rangle$ will be destroyed by the appearance of a nonzero $\delta$. In Fig. 1, we show the energy spectra and the ground states for a quantized Bose-Josephson junction with $\delta=0, E_{C}=-2.0$, and $N=20$.

For the system of a larger $N$, similar transition from nondegeneracy and degeneracy could be induced by the bifurcation. Its energy spectrum can be analyzed with the Bethe ansatz [17]. Its numerical simulation is difficult to perform; while some approximate methods including the iteration diagonalization and the density matrix renormalization group methods have been suggested, such an analysis is beyond the scope of our Letter. We stress that the physics of the analyzed situation is the source for the systems of small $(\sim 10)$ and large $\left(\geq 10^{3}\right) N$ and the used exact diagonalization is an adequate tool for our proof-ofthe-principle calculations.

By using the ground state and the first excited state as two paths of an interferometer and accomplishing the beam
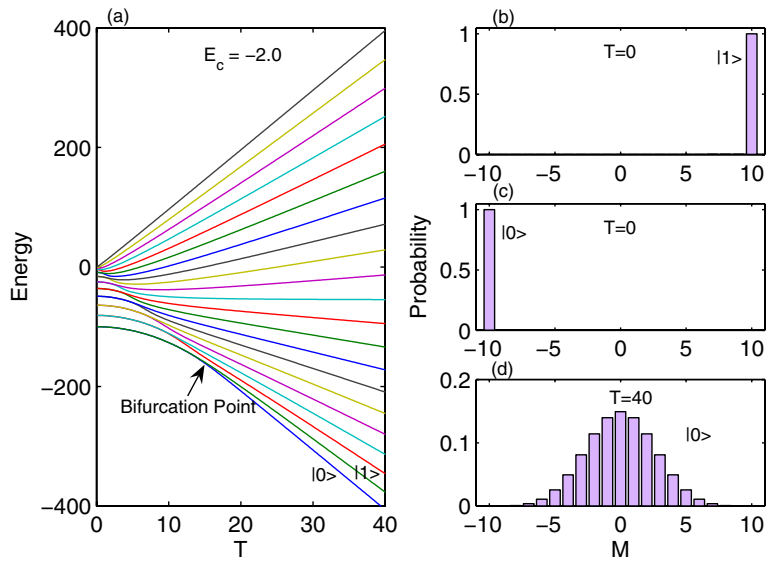

FIG. 1 (color online). The energy spectra and the ground states for a symmetric quantized Bose-Josephson junction with $E_{C}=$ -2.0 and $N=20$. (a) The energy spectra for different $T$. (b), (c) The degenerated first excited state $|1\rangle$ and the ground state $|0\rangle$ for $T=0$. (d) The ground state for $T=40$. splitting and recombination via adiabatic passage through dynamical bifurcation, it is possible to realize a MachZehnder interferometry on a quantized Bose-Josephson junction with finite negative $E_{C}$.

The first beam splitter can be achieved by preparing the ground state in the strong coupling limit and then slowly decreasing $T$ to zero, due to the appearance of a dynamical bifurcation. The negative $E_{C}$ ensures that one will get a path-entangled state $(|N / 2,-N / 2\rangle+|N / 2,+N / 2\rangle) / \sqrt{2}$ in the weak coupling limit, so that the first beam splitter also provides a route to producing a kind of entangled state. Even in the appearance of the Landau-Zener tunneling induced by the imbalance, if $|\delta|<\delta_{C}$, one can still get a path-entangled state $\left(|N / 2,-N / 2\rangle+e^{i \varphi}|N / 2,+N / 2\rangle\right) /$ $\sqrt{2}$ with a desired high fidelity. The phase difference $\varphi$ mainly comes from the imbalance and the critical value $\delta_{C}$ depends on the parameters and the desired fidelity. Utilizing the Landau-Zener tunneling as coherent beam splitters, the Mach-Zehnder interferometry has been demonstrated in a superconducting flux qubit [18]. Similarly, starting from $|N / 2,-N / 2\rangle$ or $|N / 2,+N / 2\rangle$ at $T=0$, and slowly increasing $T$ to $T \gg\left|E_{C}\right|$, beam splitting is induced by the bifurcation. In the strong coupling limit, one will obtain an equal probability superposition state of $|0\rangle$ and $|1\rangle$. In all these adiabatic processes, the evolving states perfectly keep in the sub-Hilbert space expanded by the ground state and the first excited state.

For a quantized Bose-Josephson junction with $E_{C}=$ $-2.0, N=20$, and $T=40-t$ (where $t$ is the evolution time), in Fig. 2, we show the initial state, the destination states, and the fidelities $F_{0}=|\langle 0 \mid \Psi(t)\rangle|^{2}$ and $F_{1}=$ $|\langle 1 \mid \Psi(t)\rangle|^{2}$ of the evolving state $\Psi(t)$ in a beam splitting process from $T=40$ to 0 . Here, $F_{0}$ and $F_{1}$ denote the
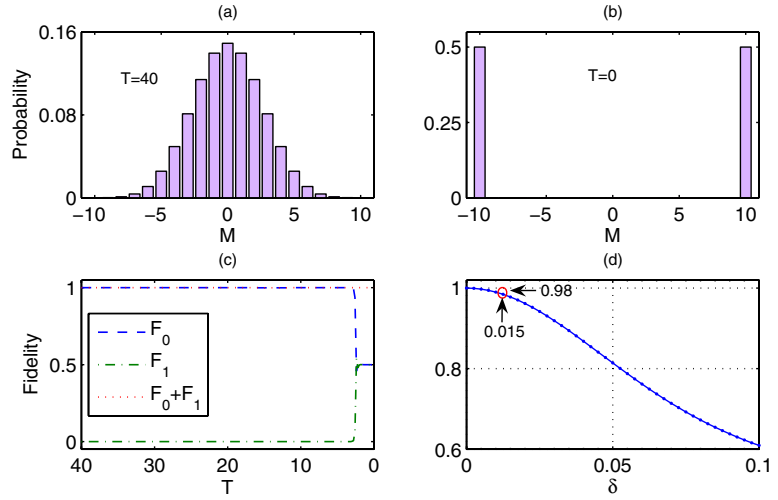

FIG. 2 (color online). The beam splitting process from $T=40$ to $T=0$ in a symmetric [cases (a-(c)] or weakly asymmetric (case d) quantized Bose-Josephson junction with $E_{C}=-2.0$ and $N=20$. (a) The initial state at $T=40$ is the ground state. (b) The destination state at $T=0$ is a path-entangled state $(|10,-10\rangle+|10,+10\rangle) / \sqrt{2}$. (c) The fidelities of the evolving states $\Psi(T)$ versus the coupling $T$. (d) The maximum fidelity $F_{\Phi}^{\max }=\max \left(|\langle\Phi(\varphi) \mid \Psi(T=0, \delta)\rangle|^{2}\right)$ of the destination state $\Psi(T=0, \delta)$ to the path-entangled state $|\Phi(\varphi)\rangle$ vs the imbalance $\delta$. 
populations of the evolving states $\Psi(t)$ occupying the states $|0\rangle$ and $|1\rangle$, respectively. The result shows the total fidelity $F_{0}+F_{1}$ almost keeps unchanged; this indicates that the environment effect is dramatically suppressed in the involved adiabatic processes. Controlling the imbalance $|\delta|<0.015$, one can get the path-entangled states $|\Phi(\varphi)\rangle=\left(|N / 2,-N / 2\rangle+e^{i \varphi}|N / 2,+N / 2\rangle\right) / \sqrt{2} \quad$ with high fidelities larger than 0.98, see Fig. 2(d).

Inducing an unknown phase shift $\phi$ between two paths with a mode-dependent force, the path-entangled state prepared by the first beam splitter evolves into a $\phi$-shifted path-entangled state $|\Phi(\phi)\rangle=(|N / 2,-N / 2\rangle+$ $\left.e^{i \phi}|N / 2,+N / 2\rangle\right) / \sqrt{2}$. To extract the information on the phase shift, one has to recombine $|\Phi(\phi)\rangle$ by the second beam splitter and monitor the populations in the two output paths. For a symmetric or a weakly asymmetric quantized Bose-Josephson junction with a negative $E_{C}$, the beam recombination can be achieved by a dynamical bifurcation or a Landau-Zener tunneling in the process of slowly increasing $T$ from 0 to $T \gg\left|E_{C}\right|$. Finally, in the strong coupling limit, the populations in the ground state and the first excited state will show interference behavior with the outcome determined by the phase shift $\phi$. In an ideal case, the fidelities of the final state to the ground and the first excited states can be exactly expressed as $\quad F_{0}=|\langle 0 \mid \Psi(T)\rangle|_{T \gg\left|E_{C}\right|}^{2}=\cos ^{2}(\phi / 2) \quad$ and $\quad F_{1}=$ $|\langle 1 \mid \Psi(T)\rangle|_{T \gg\left|E_{C}\right|}^{2}=\sin ^{2}(\phi / 2)$, respectively. This means that all particles will occupy the ground state if $\phi=2 \mathrm{k} \pi$ (where $k$ is an integer) or will stay in the first excited state if $\phi=(2 k+1) \pi$. By slowly increasing $T$ from 0 to 40 , we simulate the beam recombination process in a symmetric quantized Bose-Josephson junction with $N=20$ and $E_{C}=-2.0$ from the initial path-entangled states $|\Phi(\phi)\rangle=\left(|10,-10\rangle+e^{i \phi}|10,+10\rangle\right) / \sqrt{2}$, see Fig. 3. The fidelities of the destination state $\Psi(T=40)$ to the ground state $F_{0}$ and the first excited state $F_{1}$ show perfect behaviors of the Mach-Zehnder interference. Utilizing the

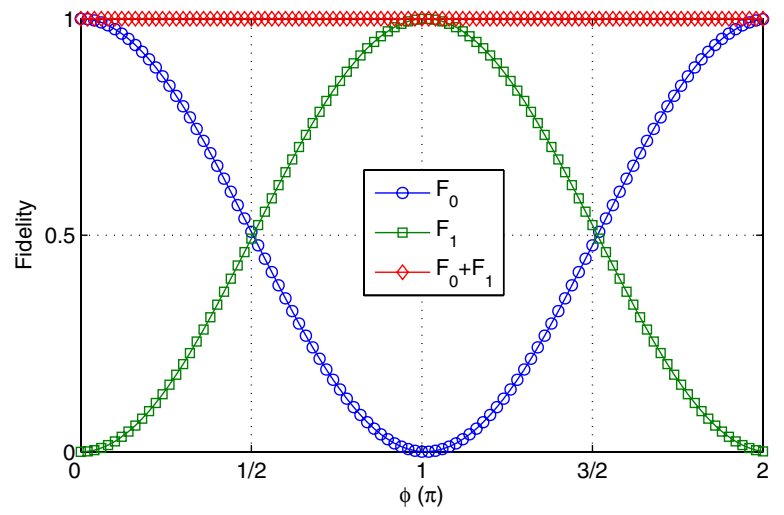

FIG. 3 (color online). Behaviors of Mach-Zehnder interference in the output states of a symmetric quantized Bose-Josephson junction with $N=20$ and $E_{C}=-2.0$ with initial states $|\Phi(\phi)\rangle$ through a dynamical bifurcation to the strong coupling limit $(T=40)$. path-entangled states in our scheme, the phase measurement precision reaches the Heisenberg limit [1]. This is in contrast to the conventional schemes using untangled single-particle states which can only reach the standard quantum limit or the shot noise limit [1].

Because of the absence of an effective method to distinguish between $|0\rangle$ and $|1\rangle$ in the strong coupling limit, it is not easy to know the populations in these two states by directly detecting the output state from the previous procedure. Fortunately, utilizing the similarity of $|0\rangle$ and $|1\rangle$ for symmetric and asymmetric quantized Bose-Josephson junctions in the strong coupling limit and the nondegeneracy of these two states for an asymmetric junction, one can distinguish these two states by suddenly applying a proper imbalance and then slowly decreasing $T$ from $T \gg\left|E_{C}\right|$ to zero (or close to zero). To determine the population of the states in the strong coupling limit, one has to avoid the dynamical bifurcation and the Landau-Zener tunneling; that is to say, one has to maintain the populations in both $|0\rangle$ and $|1\rangle$ unchanged. To avoid the dynamical bifurcation, the applied imbalance must satisfy the condition $|\delta|<$ $\left|E_{C}\right| / 2$; and to make the Landau-Zener tunneling absent, it is the best to choose the imbalance $|\delta|=\left|E_{C}\right| / 4$. Under these conditions, when $T$ approaches zero, the ground state and the first excited state become the lowest spin state $|N / 2,-N / 2\rangle$ and the highest spin state $|N / 2,+N / 2\rangle$, respectively. These two states with the largest $\left|J_{z}\right|$ correspond to all particles completely localized in either of the two modes, which can be easily detected. For an asymmetric quantized Bose-Josephson junction with $N=20$, $E_{C}=-2.0, \delta=0.5$, and $T>35$, the fidelities of the ground state and the first excited state to the symmetric counterparts are very close to 1 , i.e., $F_{00^{\prime}}=$ $\left.\left.\right|_{\delta=0}\left\langle 0^{\prime} \mid 0\right\rangle_{\delta=0.5}\right|_{T>35} ^{2} \simeq 1$ and $F_{11^{\prime}}=\left.\left.\right|_{\delta=0}\left\langle 1^{\prime} \mid 1\right\rangle_{\delta=0.5}\right|_{T>35} ^{2} \simeq$ 1, as seen in Fig. 4(b). At $T=0$, the ground state and the first excited state are the lowest spin state $|10,-10\rangle$ [see
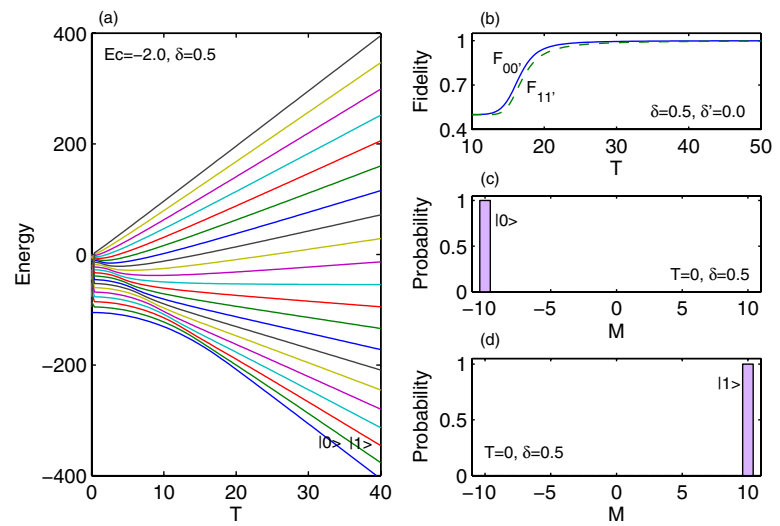

FIG. 4 (color online). The energy spectrum, the states $|0\rangle$ and $|1\rangle$, and their fidelities to the symmetric counterparts for an asymmetric quantized Bose-Josephson junction with $N=20$, $E_{C}=-2.0$, and $\delta=0.5$. (a) The energy spectrum. (b) The fidelities $F_{00^{\prime}}$ and $F_{11^{\prime}}$ for different $T$. (c) The ground state $|0\rangle$ for $T=0$. (d) The first excited state $|1\rangle$ for $T=0$. 
Fig. 4(c)] and the highest spin state $|10,+10\rangle$ [see Fig. 4(d)], respectively. Taking the output states from the second beam splitter, suddenly applying an imbalance $\delta=0.5$ and then slowly varying $T$ from 40 to 0 , our numerical simulation shows that the populations in the ground state and the first excited states remain unchanged due to the nondegeneracy and sufficiently large energy level distances.

There are two alternative approaches to the experimental realization. One possibility is to trap the condensed atoms in a double-well potential formed by external fields $[6,16]$, the other is to use a harmonically trapped two-component condensate with internal coupling between the two involved hyperfine levels $[16,19]$. In both cases, the negative charging energy can be obtained by controlling the $s$-wave scattering lengths $a_{s}$ with Feshbach resonance. For the condensates in double-well potentials, the imbalance and the coupling strength can be precisely adjusted with the techniques developed for matter-wave interference on an atom chip [6]. Assuming the experimental setup used to observe self-trapping Phys. Rev. Lett. 95, 010402 (2005) and changing $a_{s}$ from positive to negative, the parameter $\lambda=N\left|E_{C}\right| /(2 T) \sim 15$ ensures the appearance of the pathentangled state. For the coupled two-component condensates [19], the imbalance is determined by the energy difference between the two involved hyperfine levels, and can be varied by applying external magnetic fields. The coupling strength is determined by the Rabi frequency of the coupling fields, and can be controlled by changing the field intensity. Assuming the experimental setup in Phys. Rev. Lett. 81, 1539 (1998), for $N=10^{3}, \lambda=$ $N\left|E_{C}\right| /(2 T)=10$ requires the Rabi frequency $\Omega=$ $T / \hbar \sim 2 \pi \mathrm{Hz}$ and the exact path-entangled state is reached at $\Omega=0 \mathrm{~Hz}$. To count the atom numbers in a particular mode, one can use fluorescence imaging for small total numbers of atoms or use spatial imaging for large total numbers of atoms $\left(\sim 10^{3}\right.$ or larger $)$.

To conclude, we have discussed a simple and robust scheme to achieve a Heisenberg-limited Mach-Zehnder interferometry with macroscopic many-body quantum states in a quantized Bose-Josephson junction with negative charging energy, which can be realized with the present level of expertise in manipulating ultracold atoms. The interferometry not only is of fundamental physical interests, but also offers possible technological applications in high-precision measurements [1,20], in which the phase measurement precision reaches the Heisenberg limit posed by the uncertainty principle. On the other hand, given the routinely prepared condensates of $10^{3}$ or larger number of atoms, the first beam splitter in our interferometer also provides an opportunity to produce path-entangled states of very large number of particles. The presented Mach-Zehnder interferometry with path-entangled states of large number of particles has an obvious advantage over the schemes using entangled states of photons [2] or trapped ions [3], which can only operate with the order of 10 particles. All involved processes, except for the beam splitting and recombination, satisfy the adiabatic condition, and all involved quantum states are eigenstates of the system. Moreover, the sub-Hilbert space of the evolving states is closed even in procedures of beam splitting and recombination, which means that our scheme ultimately suppresses the experimental errors causing from the environment. Additionally, unlike the interferometry on linear optics, which needs one polarization detector per photon [2], our interferometry with a quantized Bose-Josephson junction needs only two detectors for any number of particles.

The author acknowledges discussions with Yuri Kivshar and Elena Ostrovskaya. This work is supported by the Australian Research Council (ARC).

*Email addresses: chl124@rsphysse.anu.edu.au; chleecn@ gmail.com

[1] V. Giovannetti, S. Lloyd, and L. Maccone, Science 306, 1330 (2004).

[2] P. Walther et al., Nature (London) 429, 158 (2004); M. W. Mitchell et al., Nature (London) 429, 161 (2004).

[3] C. A. Sackett et al., Nature (London) 404, 256 (2000); D. Leibfried et al., Science 304, 1476 (2004).

[4] M. Kitagawa and M. Ueda, Phys. Rev. A 47, 5138 (1993).

[5] M. R. Andrews et al., Science 275, 637 (1997).

[6] Y. Shin et al., Phys. Rev. Lett. 92, 050405 (2004); M. Albiez et al., Phys. Rev. Lett. 95, 010402 (2005); T. Schumm et al., Nature Phys. 1, 57 (2005).

[7] B. P. Anderson and M. A. Kasevich, Science 282, 1686 (1998); F. S. Cataliotti et al., Science 293, 843 (2001).

[8] C. Orzel et al., Science 291, 2386 (2001).

[9] A. Sørensen et al., Nature (London) 409, 63 (2001).

[10] D. Jaksch et al., Phys. Rev. Lett. 81, 3108 (1998); M. Greiner et al., Nature (London) 415, 39 (2002).

[11] S. L. Rolston and W. D. Phillips, Nature (London) 416, 219 (2002).

[12] L. Deng et al., Nature (London) 398, 218 (1999).

[13] K. E. Strecker et al., Nature (London) 417, 150 (2002); L. D. Carr and J. Brand, Phys. Rev. Lett. 92, 040401 (2004); C. Lee and J. Brand, Europhys. Lett. 73, 321 (2006).

[14] C. Lee et al., Phys. Rev. A 69, 033611 (2004).

[15] C. Lee et al., Phys. Rev. A 64, 053604 (2001); E. Shlizerman and V. Rom-Kedar, Phys. Rev. Lett. 96, 024104 (2006).

[16] S. Giovanazzi, A. Smerzi, and S. Fantoni, Phys. Rev. Lett. 84, 4521 (2000); A. Vardi and J. R. Anglin, Phys. Rev. Lett. 86, 568 (2001); S. Kohler and F. Sols, Phys. Rev. Lett. 89, 060403 (2002); G. J. Milburn et al., Phys. Rev. A 55, 4318 (1997); E. A. Ostrovskaya et al., Phys. Rev. A 61, 031601 (2000).

[17] H.-Q. Zhou et al., J. Phys. A 36, L113 (2003); V.Z. Enol'skii et al., Phys. Scr. 43, 229 (1991).

[18] W. D. Oliver et al., Science 310, 1653 (2005).

[19] C. J. Myatt et al., Phys. Rev. Lett. 78, 586 (1997); D. S. Hall et al., Phys. Rev. Lett. 81, 1543 (1998).

[20] M. A. Kasevich, Science 298, 1363 (2002). 